\title{
Three-dimensional sampling of material structure for property modeling and design
}

\author{
McLean P Echlin ${ }^{*}$, William C Lenthe and Tresa M Pollock
}

*Correspondence:
mechlin@engineering.ucsb.edu
University of California Santa
Barbara, Materials Dept. Building
503 Santa Barbara, CA, 93106-5050,
USA
USA

\begin{abstract}
Newly developed 3-D tomographic techniques permit acquisition of quantitative materials data for input to structure-property models. At the mesoscale, techniques that enable sampling of larger material volumes provide information such as grain size and morphology, 3-D interfacial character, and chemical gradients. However, systematic approaches for determining the characteristic material volume for 3-D analysis have yet to be established. In this work, the variability in properties due to microstructure is discussed in the context of a methodology for defining volume elements that link microstructure, properties, and design. As such, we propose a 3-D sampling methodology based on convergence of microstructural parameters and associated properties and design considerations.
\end{abstract}

Keywords: Representative volume element; Microstructure volume elements; Property volume elements; Femtosecond laser; Tomography; Serial sectioning

\section{Background}

With the dramatic increases in capability of 3-D tomographic techniques in the past decade [1-11], it is now possible to acquire quantitative information on material structure for higher fidelity property models. Tomographic data can be acquired across many lengthscales, using techniques such as atom probe tomography, transmission electron microscope tomography, focused ion beam serial sectioning, femtosecond laser tomography, microtomes, and both benchtop and synchrotron x-ray techniques. However, protocols for gathering 3-D data, in terms of defining representative volume elements and statistical sampling approaches, remain poorly defined for most engineering materials and their corresponding properties.

Simplified volumetric representations of materials are often made in an attempt to reduce the amount of data being passed into a component design process. Examples of these volumetric reductions include representative volume elements (RVEs) $[12,13]$, statistical volume elements (SVEs) [14,15], and statistically equivalent representative volume elements (SERVE) [16-18]. Criteria for the degree of volumetric reduction are often linked to continuum modeling assumptions, convergence of a given property, or statistical representation of specific microstructural features. Existing approaches have defined specialized RVEs that are augmented sets of sampled volume elements which are statically selected to be representative, in aggregate. Statistical representative volume element sampling has been proposed and applied [15,19-21] to materials such as titanium and fiber composites.

(c) 2014 Echlin et al.; licensee Springer. This is an Open Access article distributed under the terms of the Creative Commons Attribution License (http://creativecommons.org/licenses/by/2.0), which permits unrestricted use, distribution, and reproduction in any medium, provided the original work is properly cited. 
Engineering materials possess microstructural features across a wide spectrum of lengthscales that determine the property responses. Figure 1 shows the approximate lengthscales at which material properties (e.g., elastic modulus, creep, and fatigue) are controlled and the corresponding volume element size necessary to characterize them. The range in requisite volume element sizes is rooted in the types, sizes, and variation in the microstructures that dictate the material property responses. The property responses can be categorized as one of the following: (1) structure insensitive - properties mainly being described by the atomistic characteristic of the material, (2) dependent on the 'mean' structure - due the fact that they are dependent on the aggregate microstructural features and are relatively 'flaw insensitive', and (3) having dependence on the 'extremes' of microstructures - these are properties that depend on microstructures that are statistically rare and require very large material volume element sizes, sophisticated combinations of volume element sampling, or may require volumes entirely too large to quantify. In the case of properties such as fatigue, which may rely on very rare microstructural features, extreme value statics can be employed [22,23].

Even when employing existing structure-property models, it is still very difficult to precisely select the correct tomographic volume size for collection of experimental information that is statistically representative, can satisfy the requirements of homogenization theory, and is appropriate for the design of engineering components. We propose a method to quantitatively define volume elements that can be linked directly to microstructures/properties/design elements of interest with a finite range over which the volume element is valid.

\section{The representative volume element}

To properly describe material properties and associated constitutive response, it is necessary to select a representative volume element for analysis. Typically, RVEs are defined as

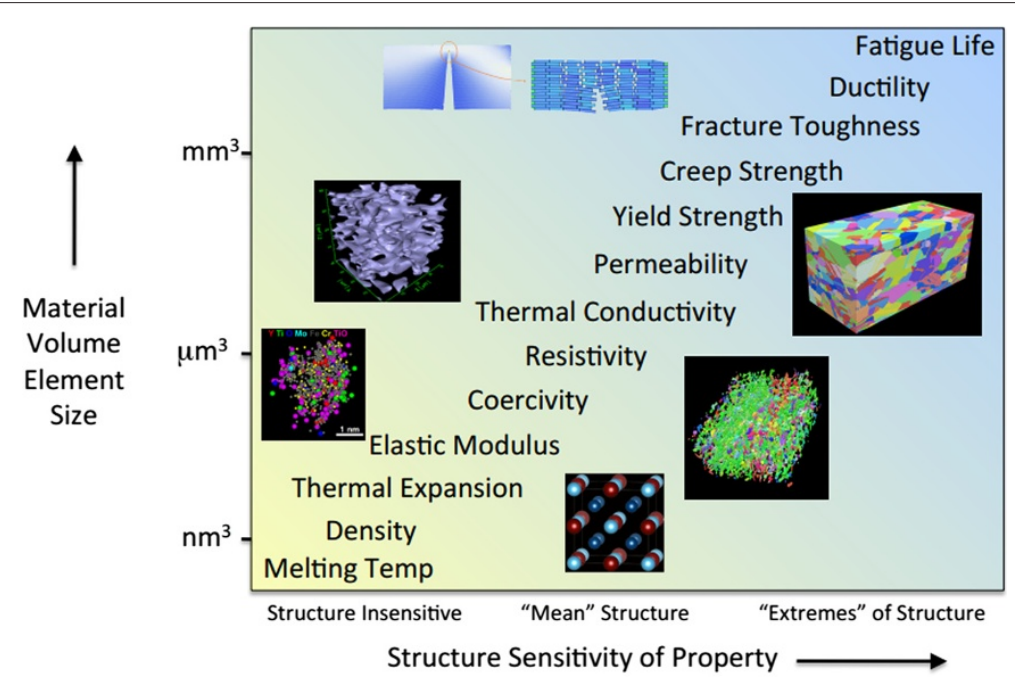

Figure 1 Material properties require differently sized material volume elements to accurately describe them. Properties can be categorized as one of three types: (1) structure insensitive - properties mainly being described by the atomistic characteristic of the material, (2) dependent on the mean structure due the fact that they are dependent on the aggregate microstructural features and are relatively flaw insensitive, and (3) having dependence on the extremes of microstructures - these are properties that depend on microstructures that are statistically rare and require very large material volume element sizes. 
a conveniently sized volume which is smaller than the macroscopic sample of interest, but large enough to be representative of the material as a whole and therefore valid for continuum homogenization assumptions. The breadth of these definitions are well summarized by Gitman [12], with most falling into one of two categories: (1) microstructure-based descriptions and (2) mechanics-based descriptors. RVEs often are defined to be of a volume that is large enough to have constitutive material properties, often only elastic properties, but small enough to be computationally tractable.

For mechanics problems, often RVEs are sized so that the Hill conditions $[13,14]$ are met. These conditions are met when the property of interest becomes independent of RVE size, according to one of the following boundary conditions: uniform displacement, uniform traction, displacement-traction, or periodic. As described by Qidwai [19], RVEs are commonly estimated by tracking property convergence as a function of increasing volume element size. They also note that the RVE dataset size often is collected and then (erroneously) used for many or all material properties. For specific applications, these definitions are useful, but not in design cases which require many material properties to be considered.

\section{Limitations of the RVE}

Continuum mechanics RVE definitions require that a volume element size randomly sampled from the bulk will have uniform material property response. Different properties have a dependence on microstructures which exist at specific length scales. For example, the elastic modulus has a strong dependence on interatomic bonding (atomic scale) and grain structure/texture (the mean structure at the mesoscale), whereas fatigue life can have a dependence on distributions of pores or other extrinsic material flaws (the extremes of structure at the mesoscale). Fatigue life would therefore require a much larger volume element to be appropriately modeled, as shown in Figure 1. It would then follow that an RVE size for the property of elastic modulus should not be used for modeling fatigue life. The discrepancies in types of RVE definitions illustrates the need for a volume element description that is better linked to the material parameters of microstructures and properties.

The RVE strategy can become complicated when the microstructures that govern the property or properties of interest span across lengthscales. In such a case, the RVE would need to be large to define mesoscale-sized features such as dendrites, grain texture, and shrinkage pores, while simultaneously having finer-scale microstructural features, such as precipitates and carbides. This strategy is problematic because it pushes the limits of the capabilities of existing tomography tools and computational methods. As such, one would prefer to decouple the fine and coarse resolution systems into separate tomography experiments. Similar types of decoupled methods have become popular with hierarchical modeling systems [24,25], although these models often only take average parameter inputs from each of the RVEs. A distinct benefit of using 3-D datasets for inputs to microstructure modeling is the ability to directly input microstructure descriptors that contain more information than single value parameters; examples of this include morphological parameters [26-28], shape parameters [29], and microstructure distribution functions [30].

New tools for gathering larger volumes of material in situ in a SEM have been developed recently $[11,31,32]$. One such tool, the TriBeam system [11], uses ultrashort femtosecond 
laser pulses to remove material through a layer-by-layer ablation process. This tool allows for the gathering of 3-D datasets either using established FIB serial sectioning [3] or by femtosecond laser ablation, which produces datasets 3 orders of magnitude volumetrically larger in time periods ranging from a few hours to a few days. The access to these substantially larger 3-D datasets motivates new approaches for probing and analyzing material volume elements. Here we use datasets generated by this new technique to address the volume element challenge.

\section{Methods}

First, we describe new volume element definitions that connect microstructure and property level descriptors with standard modes of component design. Then two sampling methods are described which (1) bound convergence criteria and the corresponding estimates of variability and (2) compare sampled volume elements from different component locations. Examples of the application of these methods will be described in 'Results' section. The ability of the new TriBeam technique to gather mesoscale-sized datasets appropriate for a range of mechanical properties is discussed.

\section{Volume element definitions}

The representative volume element is not typically clearly defined for microstructural representation, compared to property representation. As such, we define an infrastructure that connects the volume element concepts from the materials realm to the design domain, with emphasis given to building hierarchically on materials descriptors. These volume element definitions will be described presently, starting with the most fundamental microscopic descriptors and then moving up to the macroscopic scale. The most basic volume elements are defined as microstructural volume elements (MVEs), which have volumes that scale with the microstructural features of relevance. Examples of structural features that may constrain the size of the MVE include grain size, precipitate volume fraction, dendrite spacing, texture, and precipitate size. They can be defined as average quantities, distributions, or scalar quantities depending on the requirements of the structure-property models being used. Often, MVEs can be one, two, or n-point descriptors, which are covered in detail elsewhere $[21,33]$. Next, there are property volume elements (PVEs), which are linked to MVEs by existing or yet to be developed structureproperty models and therefore have sizes that scale with the microstructure volume elements on which they depend. Examples of properties that define the PVE are yield strength, elastic modulus, thermal conductivity, and permeability, which will be discussed as example cases in 'Results' section. Contrary to intuition, PVEs are not simply defined as the maximum size of their dependent MVEs; this will be more rigorously addressed later in the paper. The design volume element (DVE) is composed of the volume of an engineering component being designed or alternatively as a sub-region of the component of interest. For example, an engineering component may be designed to remain elastic over its entire volume, except for a small volume of material located adjacent to a stressconcentrating notch. In this event, the MVE and PVE for the elastic modulus would apply to the bulk of the component and, for example, the yield strength PVE would then be used to size the notch. Therefore, the DVE is specific to the component and its anticipated application. A schematic of the relations of the MVE/PVE/DVEs is shown in Figure 2, with example structure-property models inset within the connectivity between MVE and PVE, and example MVE and PVE definitions labeled within the boxes. 


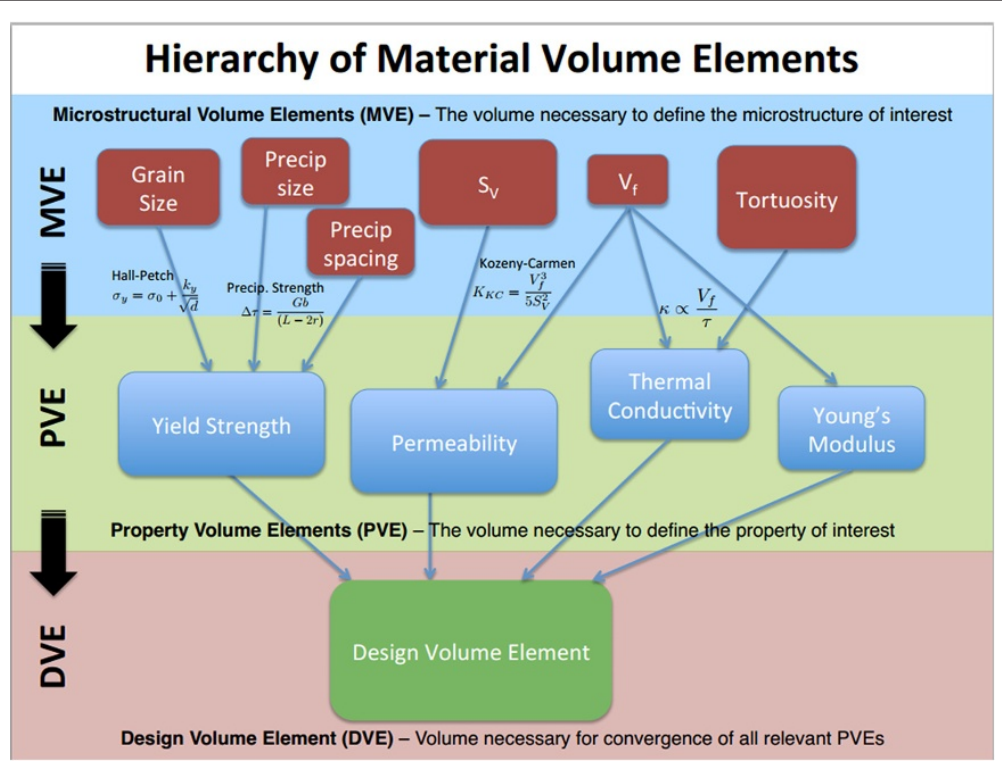

Figure 2 Material volume elements can be divided into a hierarchy which is tiered based on their dependence. On the top tier, examples of selected MVE are shown with their relative expected sizes (conveyed by box size). On the middle tier are PVEs, which have dependence on MVEs. Next to the arrows indicating the dependencies are the structure-property relations which model the expected relationship between the MVEs and the PVEs. Design volume elements are displayed on the lowest level. A DVE is defined for specific PVEs, over which it has been validated for by means of property convergence. The DVE size will be determined based on the geometric effects of the components that are being designed.

The volume element definitions presented in this section can be used in the following two ways: (1) a DVE volume can be defined as the size at which point all MVEs and PVEs converge; (2) in the event that the DVE is instead limited by the physical design constrains of the part, then alternately the variability of the MVEs and PVEs applicable to the design problem can be assessed to provide information for the minimum material property design limits. In other words, the DVE size limits the problem and therefore the variability in PVEs at the prescribed size can be evaluated. Examples of these methods will be given in the 'Results' section.

\section{Sampling for convergence size}

Two distinct materials sampling methods are presented to define volume elements for MVEs or PVEs. The first method is used to randomly sample $n$ volumes of equal size across a range of increasing volume sizes $\left(V_{1}, V_{2}, \ldots, V_{i}\right)$ in order to determine the volume $V_{\mathrm{c}}$ at which microstructure or property convergence occurs, shown in Figure 3 . This method has been applied to materials such as tungsten copper $(\mathrm{WCu})$ composite, using the MVEs of volume fraction $\left(V_{\mathrm{f}}\right)$ and surface area to volume ratio $\left(S_{\mathrm{v}}\right)$ for the PVEs of permeability $(K)$, and polycrystalline Young's modulus $(E)$ which is shown in more detail in 'Results' section and in [34]. Convergence of these MVEs or PVEs was determined using a standard $99 \%$ confidence interval bound (to be within $5 \%$ of the mean), shown in Figure 4, and applying statistical hypothesis tests, such as the $t$ test and $z$ test. For example, the $t$ test confidence interval (CI) around the sampled volume fraction average, $\overline{V_{\mathrm{f}}}$, is defined as

$$
\mathrm{CI}=\overline{V_{\mathrm{f}}} \pm t^{*} \frac{\sigma_{V_{\mathrm{f}}}}{\sqrt{n}}
$$



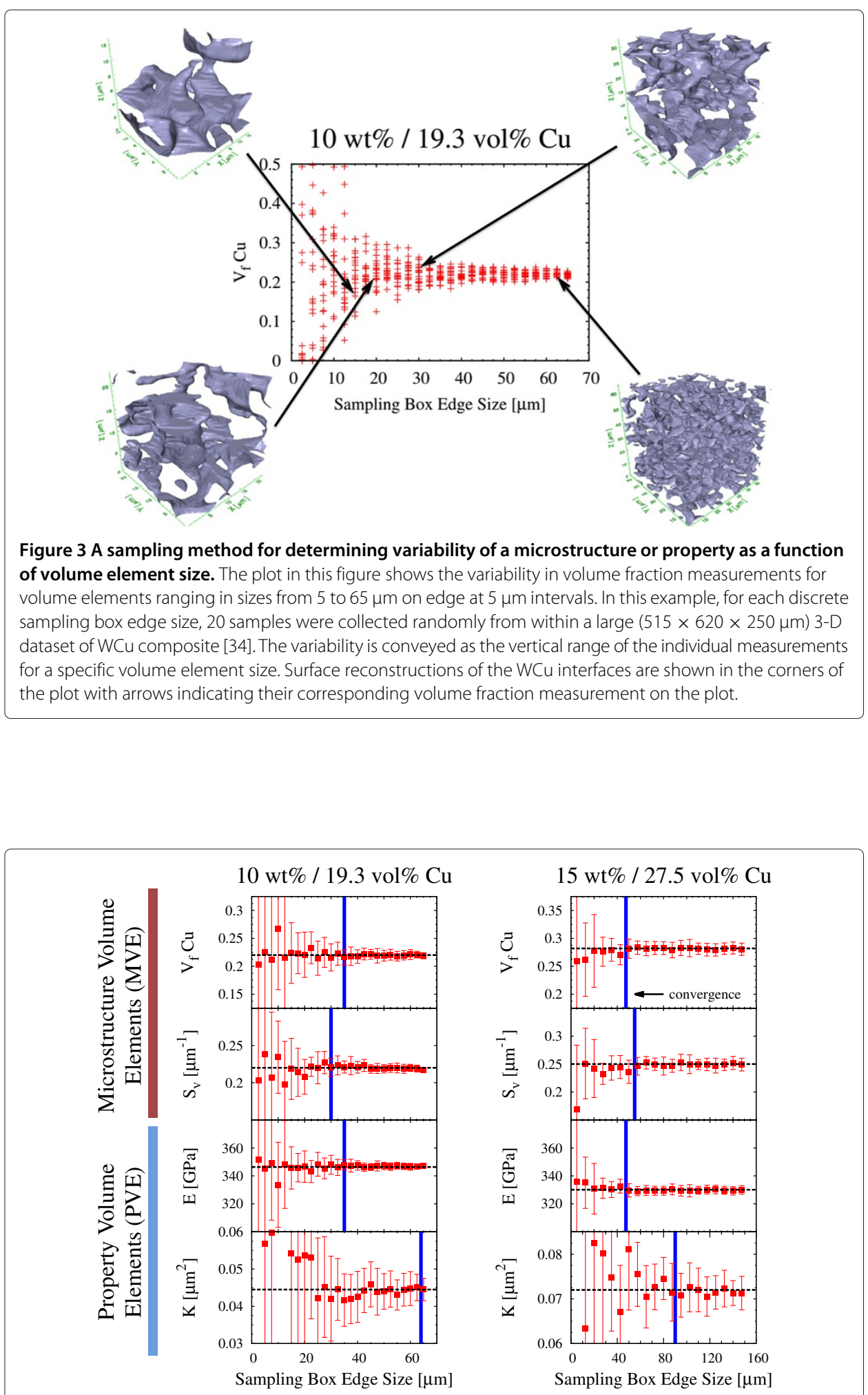

Figure 4 Plots of microstructural and property descriptors are shown for a WCu composite. The measured microstructure parameters are surface area to volume ratio $\left(S_{v}\right)$ and volume fraction $\mathrm{Cu}\left(V_{f}\right)$. The properties calculated are elastic modulus ( $E$ ) using the rule of mixtures and permeability $(K)$ using the Kozeny-Carmen relation (see Equation 2). Convergence of each property is shown for a $99 \%$ confidence interval, which is explained in more detail elsewhere [34]. The variability in $K$ is greater than $E$, due to its dependence on both $V_{f}$ and $S_{v}$. 
where $\sigma_{V_{\mathrm{f}}}$ is the sampled standard deviation in $\mathrm{V}_{\mathrm{f}}, t^{*}$ is evaluated from the $t$ distribution for the desired confidence index, and $n$ is the number of randomly sampled volumes. Furthermore, the confidence interval can be expressed in terms of the coefficient of variation $\left(C_{\mathrm{v}}=\sigma_{V_{\mathrm{f}}} / \overline{V_{\mathrm{f}}}\right)$. This general methodology of tracking convergence has been used regularly in problems such as random composites [35], ice cream [36], hydrided Zircaloy cladding [37], and the WCu composite discussed in this research.

\section{Sampling for rare or site-specific structural features}

The second method for sampling is more relevant for properties that require volume element sizes which are inaccessible due to a mismatch between the capabilities of tomography and the volume to be sampled or require sampling from site-specific locations. Examples of rare features that can be interrogated include interconnect defects in electronics components and porosity in cast metals. Examples of site-specific microstructural features include grain boundaries or specially oriented crystals. Using a tool such as the TriBeam [11] or a dual-beam focused ion beam (FIB), targeted dataset acquisitions can be made from multiple locations within a sample, shown schematically in Figure 5 .

It is often of interest to collect datasets which are spatially located near design features which are known to accelerate events leading to failure or be deleterious to local material properties. Stress concentrators such as notches or cracks are particularly detrimental under fatigue and static loads [38] where the local structure and properties are of strong interest. In such cases, one would like to measure the microstructure features and local properties nearby the geometric irregularity that will be preferentially sampled by the design feature. It is also important to consider cases where the design-imposed geometric constraints interrogate a volume that is much smaller than the microstructure or property volume elements.

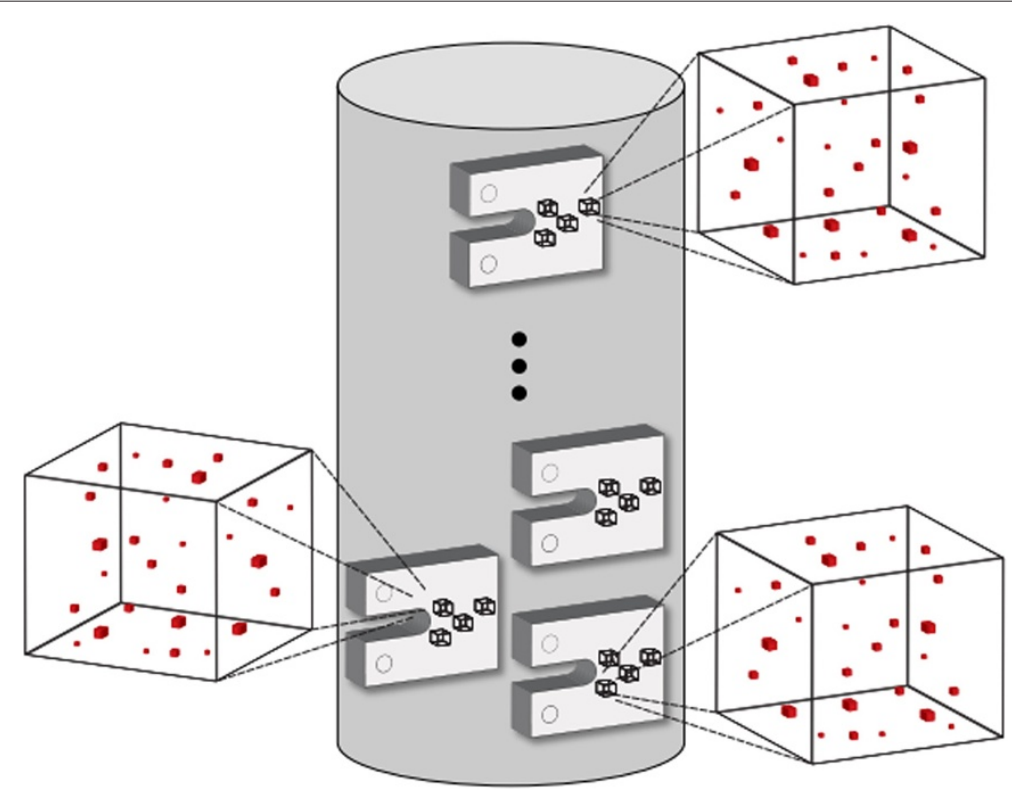

Figure 5 Sampling method for use with extremely large property or microstructure volume elements (MVEs or PVEs). A component can be randomly (or strategically) sampled to find long range variation in microstructure or to capture 'rare' features. In this methodology, a volume element size may be chosen that is not fully converged but sampled often enough to define the rare events. 
This method of sampling for rare structures was employed to gather large 3-D datasets from a high-strength steel containing widely spaced titanium nitride (TiN) inclusion phases that have sizes ranging from 1 to $10 \mu \mathrm{m}$ at volume fractions of $0.01 \%$ to $0.05 \%$. During crack advance, the TiN inclusions are deleterious to fracture toughness, a material property which has a large PVE. TiN 3-D datasets were gathered from many locations on a compact tension sample, collecting different plastic zone-sized volumes that a crack tip would sample during crack propagation [39]. These datasets were then used, in aggregate, to measure the various spatial distributions of TiN inclusions in a highstrength steel and assess how the TiN inclusions contribute to the variability in fracture toughness.

\section{Results}

The MVE/PVE/DVE hierarchy has been designed to connect the existing structureproperty relations (models) with component design architecture. In the following sections, we demonstrate its use for several sample systems with 3-D data gathered by femtosecond laser-aided tomography in both the vacuum chamber (TriBeam) and in ambient laboratory air with optical imaging.

\section{MVE and PVE variability}

WCu composite datasets were collected using the TriBeam [11] in less than $48 \mathrm{~h}$ with volumes as large as $615 \times 525 \times 250 \mu \mathrm{m}$ with a 250 -nm slice thickness [34]. The TriBeam uses a femtosecond laser to ablate sections of material at rates that are 4 to 5 orders of magnitude faster than the standard focused ion beam source available in many dual-beam FIB microscope system. The resulting image stacks from the tomography experiments are composed of $100 \mathrm{~s}$ to $1000 \mathrm{~s}$ of secondary electron images, which were segmented, registered, and reconstructed in 3-D.

Two different composition WCu datasets were collected using the TriBeam system [34] and sampled to analyze the convergence of MVEs and PVEs, shown in Figure 4. Samples were collected by randomly selecting volumes at $5 \mu \mathrm{m}$ intervals between 5 and $65 \mu \mathrm{m}$ on the edge for a W-10 wt.\% Cu composite dataset and 5 to $160 \mu \mathrm{m}$ on edge for a W$15 \mathrm{wt} . \% \mathrm{Cu}$ composite dataset. A total of 20 random samples were taken for each volume in order to calculate the variability in two microstructural parameters and two material properties. A sensitivity study was performed to determine the number of samples necessary for variability analysis and the results are shown in Figure 6. These data show that for $n$ (number of randomly sampled volume elements) greater than 5 to 10, variability plateaus; therefore, all analyses were performed at 20 samples per volume. Figure 4 shows the average value of each of 20 samples plotted as solid squares, for both W-10 wt.\% Cu and W-15 wt.\% Cu composites, with the standard deviations in the sample sets indicated with bar lines for volume fraction $\left(V_{\mathrm{f}}\right)$, surface area to volume ratio $\left(S_{\mathrm{v}}\right)$, permeability $(K)$, and polycrystalline effective elastic modulus $(E)$. The microstructural or property average values that the data are converging toward are shown as a horizontal dotted line, while the converged volume element size (with $99 \%$ confidence interval to be within $5 \%$ of the mean) is shown as a vertical solid line. The MVEs $\left(V_{\mathrm{f}}\right.$ and $\left.S_{\mathrm{v}}\right)$ converge faster than the PVEs $(K$ and $E)$, as illustrated by the positions of the vertical lines in Figure 4. Also, the aggregate-converged PVE size is non-intuitively larger than the largest dependent MVE convergence size. This result demonstrates the compounding variability that accrues with 
$10 \mathrm{wt} \% / 19.3 \mathrm{vol} \% \mathrm{Cu}$

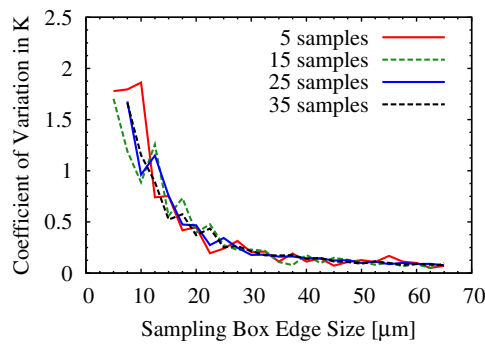

$15 \mathrm{wt} \% / 27.5 \mathrm{vol} \% \mathrm{Cu}$

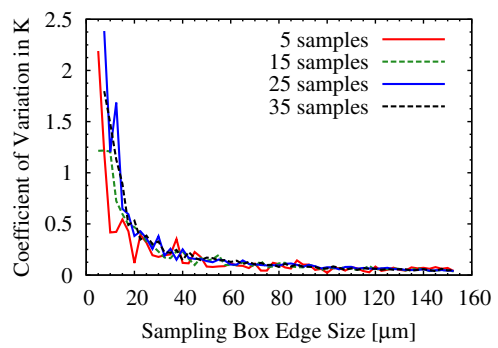

Figure 6 Unit of normalized variability, coefficient of variation for $\boldsymbol{K}$. Plots showing a unit of normalized variability, coefficient of variation ( $C V=\frac{\sigma_{\text {sample }}}{\mu_{\text {sample }}}$, for calculated permeability $(K)$ as a function of sampling box size for sampled sets of size $n=5,15,25$, and 35. Two WCu composite materials are shown, 10 and $15 \mathrm{wt} . \%$ $\mathrm{Cu}$. The number of samples collected at each volume element box size has little effect on the variability above $n=5$ or 10 samples.

multiple MVE dependence of a PVE. Linking the predicted MVE variability at a specified PVE size will be shown to be a valuable tool in 'Discussion' section.

Error analysis, which is well detailed elsewhere [40], can be also be performed for many existing analytical structure-property models to determine the uncertainty in a sampled property average. For example, the variability in permeability $(K)$, as defined by the Kozeny-Carmen relation,

$$
K=\frac{V_{f}^{3}}{5 S_{V}^{2}}
$$

where $V_{\mathrm{f}}$ is the volume fraction $\mathrm{Cu}$, and $S_{\mathrm{V}}$ is the surface area to volume ratio which can be represented as

$$
\sigma_{K}=\sqrt{\left(\frac{\partial K}{\partial V_{\mathrm{f}}}\right)^{2} \sigma_{V_{\mathrm{f}}}^{2}+\left(\frac{\partial K}{\partial S_{\mathrm{V}}}\right)^{2} \sigma_{S_{\mathrm{V}}}^{2}+2 \frac{\partial K}{\partial S_{\mathrm{V}}} \frac{\partial K}{\partial V_{\mathrm{f}}} \sigma_{S_{\mathrm{V}} V_{\mathrm{f}}}}
$$

where $\sigma$ terms represent standard deviation and $\sigma_{S_{\mathrm{V}} V_{\mathrm{f}}}$ is the covariance of the two MVEs. In Figure 7, the uncertainty has been plotted with and without the covariance term, $\sigma_{S_{\mathrm{V}} V_{\mathrm{f}}}$, which is only necessary for a PVE (e.g., $K$ ) that has MVEs that are codependent (e.g., $V_{\mathrm{f}}$ and $S_{\mathrm{V}}$ ). Notably, the uncertainty calculation with the covariance term included has a better fit with the sampled data volume, suggesting that the MVEs of $V_{\mathrm{f}}$ and $S_{\mathrm{V}}$ are both dependent on similar microstructural features and lengthscales, which is more clearly shown in the standard deviation plots of the same three calculations in Figure 8. Therefore, error analysis can be used to estimate PVE sizes from MVEs where the structure-property relation is analytically defined, whereas the PVE size must be determined by direct measurements of variability in the property from sampled volumes in all other cases. For example, error analysis requires a different approach in more complicated structure-property modeling relations such as numerical simulations of fluid flow or plasticity models that rely on non-analytical solutions or have stochastic components.

\section{Design volume elements}

Design volume elements (DVEs) are component specific. Consider for example the use of the WCu composite in a non-structural thermal protection application where ablative cooling via vaporization of $\mathrm{Cu}$ is required. Assuming stresses are thermal and well below 


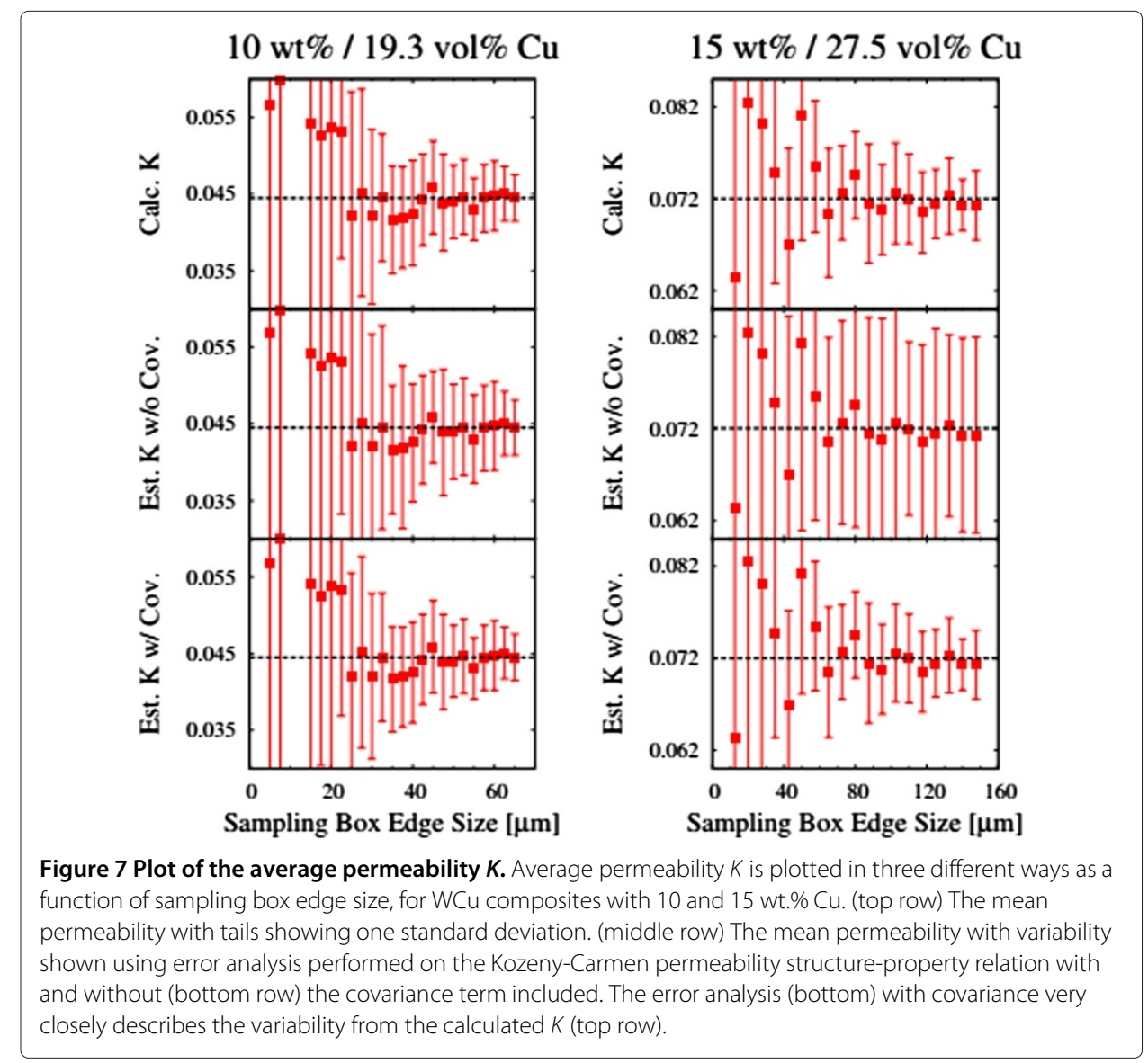

the yield strength of the tungsten phase, the two primary properties of interest would have elastic modulus and permeability.

A volume element dependency chart similar to Figure 2 can be constructed for the DVE for the described thermal protection application, shown in Figure 9. This example shows the magnitude dependence in the amount of variability in the elastic modulus as

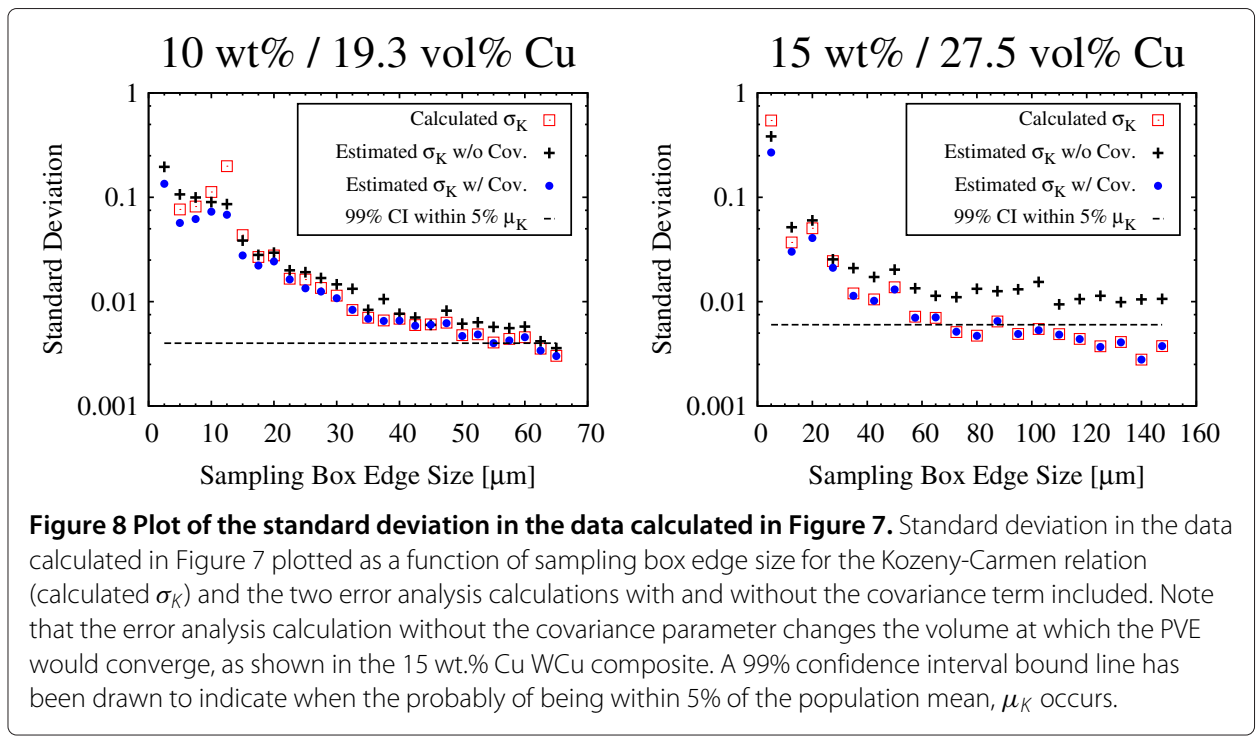




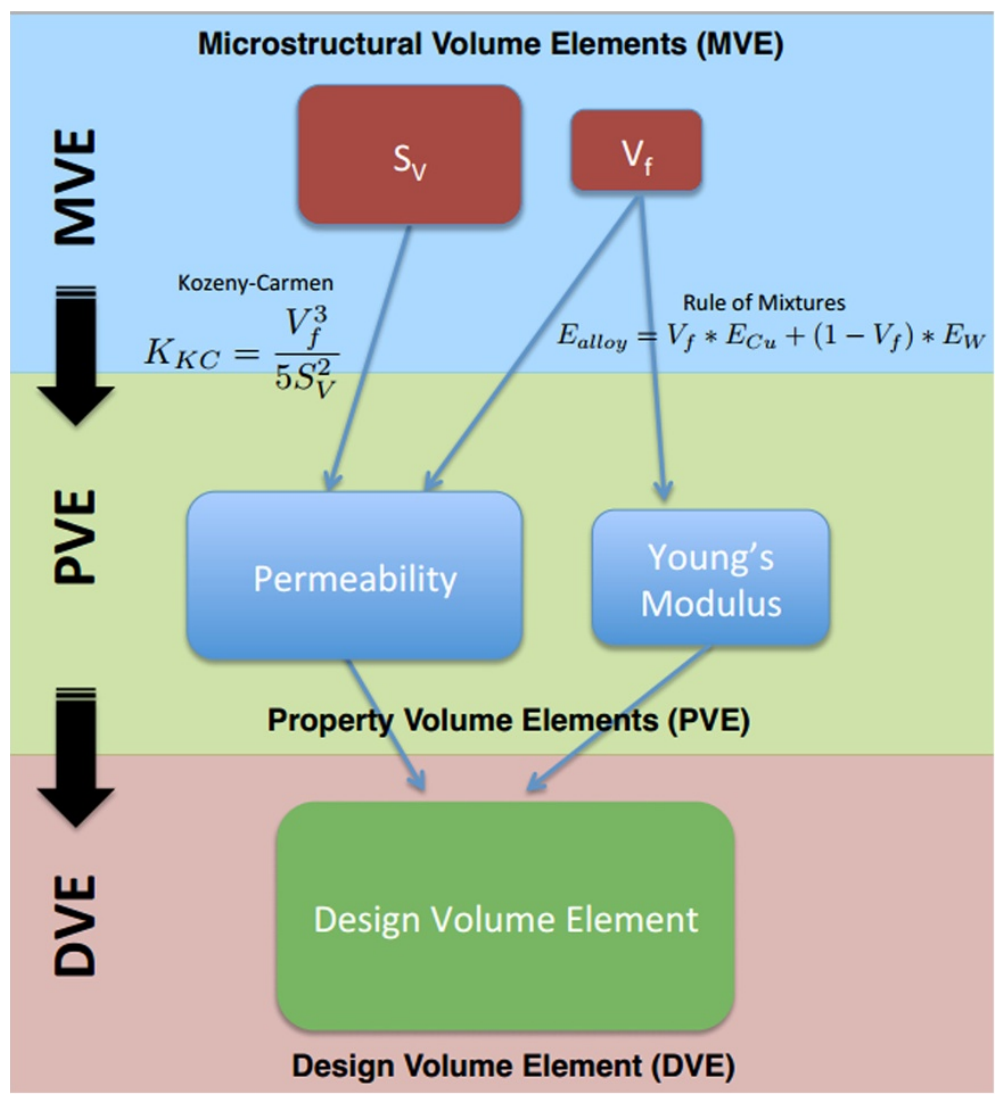

Figure 9 Hypothetical DVE hierarchy. A hypothetical DVE hierarchy constructed for a WCu composite that would be applicable for an ablatively cooled thermal protection application. The variability of each PVE is compounded by the type of dependencies it has on MVEs. In this example, permeability $K$ is dependent on $V_{f}$ and $S_{V}$ through the Kozeny-Carmen relation and $E$ is dependent on $V_{f}$ by the rule of mixtures.

a function of $V_{\mathrm{f}}$ and the variability in permeability with both $V_{\mathrm{f}}$ and $S_{\mathrm{v}}$. In this application, a DVE of size greater than the combined variability of both the elastic modulus and permeability will be necessary. Using the convergence data shown in Figure 4, then the DVE volume required for the W-10 wt.\% Cu composite would be $>65 \mu \mathrm{m}$ on edge and the W-15 wt.\% Cu composite volume would be $>90 \mu \mathrm{m}$ on edge for convergence in both properties. However, if a smaller volume is selected, the variability of the properties can be determined for the specified volume as indicated by the bar widths in Figures 3 and 4. For example, if a DVE was determined to be $60 \mu \mathrm{m}$ on edge in the W- $15 \mathrm{wt} . \% \mathrm{Cu}$ dataset, then 1 standard deviation from the mean ranges between 0.064 and 0.078 , with a minimum permeability of 0.05 . One can imagine that a larger set of properties would be required in other applications, i.e., yield strength, elastic modulus, fatigue strength, and permeability in this event the DVE would likely be even larger.

\section{Discussion}

RVEs used in design often assume uniform material properties and typically have sizes based only on mechanics considerations. However, to predict properties with a prescribed degree of confidence, it is important to account for the distribution of each of the microstructural features that influence the property of interest. Therefore, microstructural variability, shown in Figure 10, must be included in the property calculation in 


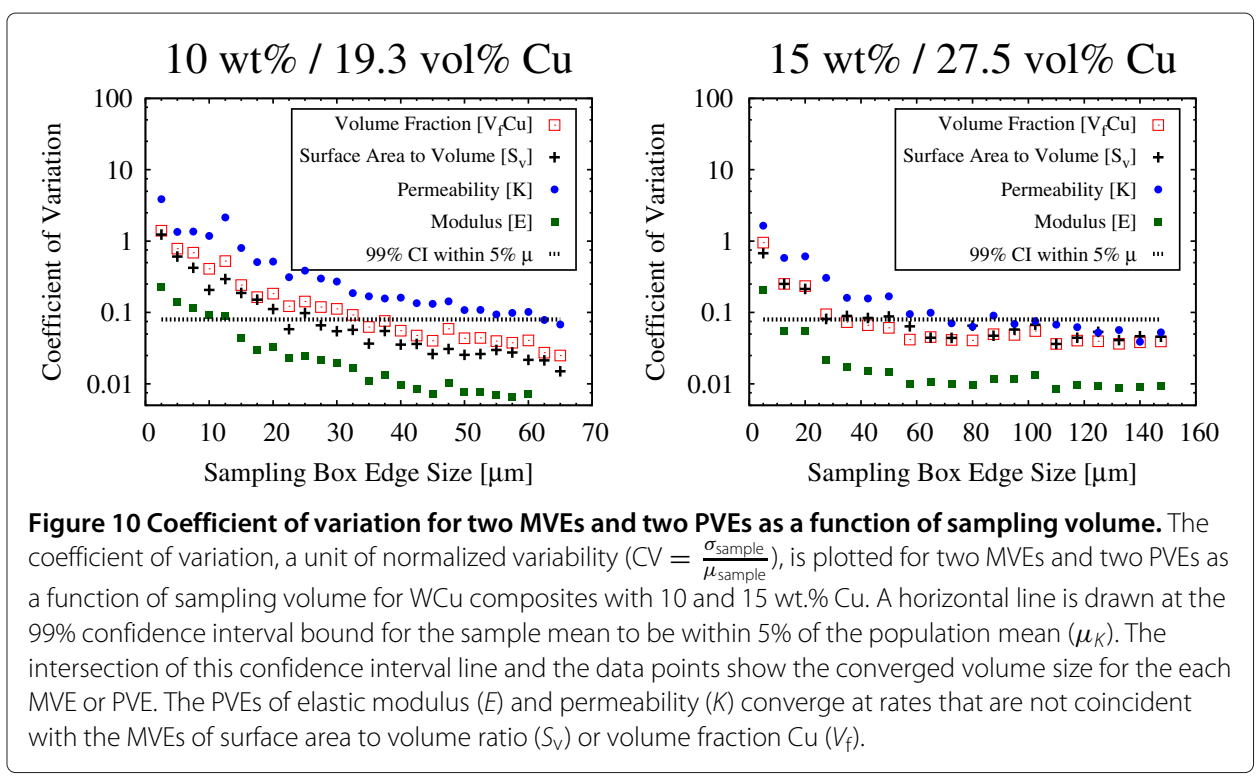

order to correctly predict the variability in material response and the lower bounds of the property. For analytically defined structure-property models such as the permeability PVE, shown in 'Results' section, convergence can be predicted using error analysis (see Equation 3). Furthermore, variability in microstructure must also be considered when specific design geometries are introduced, such as notches, which will create higher localized stresses. Mechanics calculations which have included material properties have been applied in works such as those by Lazzarin and Berto [38], where the material toughness was incorporated with the standard notched crack tip strain field calculation in order to resolve fatigue life calculations. We assert that models such as this could be enhanced with the use of converged material and property volume element calculations, which can define a range of variability in material parameters that can be directly input to mechanics calculations such as these.

Large 3-D datasets are often either not computationally tractable or collection is not feasible experimentally. In either of these situations where the microstructure or property volume element (MVE or PVE) may not have converged, statistical analysis can be applied. For average properties, one can apply distribution assumptions (typically Gaussian) and predict the variability expected for the non-converged dataset. For example, if a dataset was gathered at a volume smaller than the converged sizes shown in Figure 4, then the analysis shown in 'Sampling for convergence size' section can be applied with an assumption of a 99\% (or other) confidence interval to predict the approximate volume necessary for convergence. Furthermore, the expected variability in the measured microstructure or property parameters at the collected dataset volume element size can be inferred from the confidence interval bounds. However, this methodology will not be predictive for properties that rely on extreme value microstructure descriptors, such as fatigue, except in cases where the microstructural distribution has been well characterized [20,23,41-43]. Finally, we note that the rapid advancement of 3-D tomography techniques will increasingly enable the collection of microstructural datasets of large enough volumes to properly bound material 
properties as well as enable the development of improved property models for a range of materials systems.

\section{Conclusions}

Based on the above results, we have derived the following:

- A method for categorizing and quantifying volume elements based on microstructure, properties, and design has been presented (MVE, PVE, and DVE).

- Sampling methods for determining convergence of MVEs and PVEs are presented for the case of a WCu 3-D dataset.

- PVEs converge (variability decreases to a specified confidence interval) at rates that are greater than those of their MVE dependencies.

- MVEs and PVEs converge at different rates and sizes; therefore, a volume element should only be used to model properties for which it has been validated.

- Microstructural volume elements that are smaller than the converged size can be useful to calculate the expected variability for that volume.

Competing interests

The authors declare that they have no competing interests.

\section{Author's contributions}

MPE performed the datasets acquisitions and reconstructions and analysis. WCL provided statistical analysis and convergence criteria. TMP participated in the design and coordination of this work through its entirety. All authors read and approved the final manuscript.

\section{Acknowledgements}

The authors acknowledge the Office of Naval Research ONR-DURIP grant no. N000141010783 for the support of the development of the TriBeam system and Air Force grant FA9550-12-1-0445 for the support of this research. We also thank FEl Corporation for supporting the development of the TriBeam system. The authors acknowledge David Rowenhorst for visualization code in IDL and Michael Uchic for useful discussions on tomography. The thoughtful comments from the reviewers are also greatly appreciated.

Received: 31 December 2013 Accepted: 30 May 2014

Published: 27 June 2014

\section{References}

1. Midgley PA, Weyland M (2003) 3D electron microscopy in the physical sciences: the development of z-contrast and $\{$ EFTEM tomography In: Proceedings of the international workshop on strategies and advances in atomic level spectroscopy and analysis. Ultramicroscopy. (3-4):413-431. doi:10.1016/S0304-3991(03)00105-0

2. Holzer L, Indutnyi F, Gasser P, Munch B, Wegmann M (2004) Three-dimensional analysis of porous $\mathrm{BaTiO}_{3}$ ceramics using FIB, nanotomography. J Microsc 216(1):84-95. doi:10.1111/j.0022-2720.2004.01397.x

3. Uchic MD, Groeber MA, Dimiduk DM, Simmons JP (2006) 3D microstructural characterization of nickel superalloys via serial-sectioning using a dual beam FIB-SEM In: Viewpoint set no. 4: 3D characterization and analysis of materials. Organized by G. Spanos. Scripta Mater 55(1):23-28. doi:10.1016/.jscriptamat.2006.02.039

4. Jensen DJ, Poulsen HF (2012) The three dimensional x-ray diffraction technique. Mater Char 72(0):1-7. doi:10.1016/..matchar.2012.07.012

5. Suter RM, Hennessy D, Xiao C, Lienert U (2006) Forward modeling method for microstructure reconstruction using x-ray diffraction microscopy: single-crystal verification. Rev Sci Instrum 77(12). doi:10.1063/1.2400017

6. Landis EN, Keane DT (2010) X-ray microtomography. Mater Char 61(12):1305-1316. doi:10.1016/j.matchar.2010.09.012

7. Alkemper J, Voorhees PW (2001) Quantitative serial sectioning analysis. J Microsc 201(3):388-394. doi:10.1046/j.1365-2818.2001.00832.x

8. Spowart J, Mullens H, Puchala B (2003) Collecting and analyzing microstructures in three dimensions: a fully automated approach 55(10):35-37. doi:10.1007/s11837-003-0173-0

9. Rowenhorst DJ, Lewis AC, Spanos G (2010) Three-dimensional analysis of grain topology and interface curvature in a beta-titanium alloy. Acta Mater 58(16):5511-5519. doi:10.1016/.actamat.2010.06.030

10. Uchic M, Groeber M, Spowart J, Shah M, Scott M, Callahan P, Shiveley A, Chapman M (2012) An automated multi-modal serial sectioning system for characterization of grain-scale microstructures in engineering materials (preprint). Technical report, DTIC Document

11. Echlin MP, Mottura A, Torbet CJ, Pollock TM (2012) A new TriBeam system for three-dimensional multimodal analysis. Rev Sci Instrum 83(2):doi:10.1063/1.3680111

12. Gitman IM, Askes H, Sluys LJ (2007) Representative volume: existence and size determination. Eng Fract Mech 74(16):2518-2534. doi:10.1016/j.engfracmech.2006.12.021

13. Hill R (1963) Elastic properties of reinforced solids: some theoretical principles. J Mech Phys Solid 11(5):357-372. doi:10.1016/0022-5096(63)90036-X

14. Ostoja-Starzewski M (2006) Material spatial randomness: from statistical to representative volume element Probabilist Eng Mech 21(2):112-132. doi:10.1016/j.probengmech.2005.07.007 
15. Niezgoda SR, Turner DM, Fullwood DT, Kalidindi SR (2010) Optimized structure based representative volume element sets reflecting the ensemble-averaged 2-point statistics. Acta Mater 13:4432-4445. doi:10.1016/j.actamat.2010.04.041

16. Groeber M, Haley BK, Uchic MD, Dimiduk DM, Ghosh S (2006) 3D reconstruction and characterization of polycrystalline microstructures using a FIB-SEM system. Mater Char 57:259-273. doi:10.1016/j.matchar.2006.01.019

17. Swaminathan S, Ghosh S, Pagano N (2006) Statistically equivalent representative volume elements for unidirectional composite microstructures: part i - without damage. J Compos Mater 40(7):583-604

18. Shan Z, Gokhale AM (2002) Representative volume element for non-uniform micro-structure. Comput Mater Sci 24(3):361-379. doi:10.1016/S0927-0256(01)00257-9

19. Qidwai SM, Turner DM, Niezgoda SR, Lewis AC, Geltmacher AB, Rowenhorst DJ, Kalidindi SR (2012) Estimating the response of polycrystalline materials using sets of weighted statistical volume elements. Acta Mater 60:5284-5299. doi:10.1016/j.actamat.2012.06.026

20. Groeber M, Ghosh S, Uchic MD, Dimiduk DM (2008) A framework for automated analysis and simulation of 3d polycrystalline microstructures. part 2: synthetic structure generation. Acta Mater 56(6):1274-1287

21. McDowell DL, Ghosh S, Kalidindi SR (2011) Representation and computational structure-property relations of random media. JOM 63(3):45-51. doi:10.1007/s11837-011-0045-y

22. Brundidge CL, Pollock TM (2012) Processing to fatigue properties: benefits of high gradient casting for single crystal airfoils. In: Huron ES, Reed RC, Hardy MC, Mills MJ, Montero RE, Portella PD, Telesman J (eds) Superalloys 2012. Wiley, Hoboken, pp 379-385. doi:10.1002/9781118516430.ch41. http://dx.doi.org/10.1002/9781118516430.ch41

23. Przybyla CP, McDowell DL (2010) Microstructure-sensitive extreme value probabilities for high cycle fatigue of Ni-base superalloy \{IN100\}. Int J Plast 26(3):372-394. doi:10.1016/j.jplas.2009.08.001

24. Vernerey F, Liu WK, Moran B, Olson G (2009) Multi-length scale micromorphic process zone model. Comput Mech 44(3):433-445

25. McDowell DL (2010) A perspective on trends in multiscale plasticity. Int J Plast 26(9):1280-1309

26. Rowenhorst D, Kuang J, Thornton K, Voorhees P (2006) Three-dimensional analysis of particle coarsening in high volume fraction solid-liquid mixtures. Acta mater 54(8):2027-2039

27. Madison J, Spowart J, Rowenhorst D, Pollock T (2008) The three-dimensional reconstruction of the dendritic structure at the solid-liquid interface of a Ni-based single crystal. JOM 60(7):26-30

28. Rowenhorst D, Voorhees P (2012) Measurement of interfacial evolution in three dimensions. Annu Rev Mater Res 42:105-124

29. MacSleyne J, Uchic MD, Simmons JP, Graef MD (2009) Three-dimensional analysis of secondary $\gamma$ / precipitates in Rene-88 \{DT\} and UMF-20 superalloys. Acta Mater 57(20):6251-6267. doi:10.1016/j.actamat.2009.08.053

30. Groeber M, Ghosh S, Uchic MD, Dimiduk DM (2008) A framework for automated analysis and simulation of 3D polycrystalline microstructures.: part 1: statistical characterization. Acta Mater 56(6):1257-1273. doi:10.1016/j.actamat.2007.11.041

31. Taklo MM, Klumpp A, Ramm P, Kwakman L, Franz G (2011) Bonding and TSV in $3 D$ IC integration: physical analysis with plasma FIB. Microsc Anal 25(7):9-12

32. Altmann F, Beyersdorfer J, Schischka J, Krause M, Franz G, Kwakman L (2012) Cross section analysis of Cu filled TSVS based on high throughput plasma-FIB milling In: ISTFA, 2012: ASM international conference proceedings of the 38th international symposium for testing and failure analysis. ASM International, Geauga County, pp 39-43

33. Torquato S (2002) Random heterogeneous materials: microstructure and macroscopic properties. Interdisciplinary applied mathematics: mechanics and materials, vol. 16.. Springer, New York. http://books.google.com/books?id= PhG_X4-8DPAC

34. Echlin MP, Mottura A, Wang M, Mignone PJ, Riley DP, Franks GV, Pollock TM (2013) Three-dimensional characterization of the permeability of W-Cu composites using a new "Tribeam" technique. Acta Materi 64:307-315. doi:10.1016/j.actamat.2013.10.043

35. Kanit T, Forest S, Galliet I, Mounoury V, Jeulin D (2003) Determination of the size of the representative volume element for random composites: statistical and numerical approach. Int J Solid Struct 40:3647-3679. doi:10.1016/S0020-7683(03)00143-4

36. Kanit T, N'Guyen F, Forest S, Jeulin D, Reed M, Singleton S (2006) Apparent and effective physical properties of heterogeneous materialsrepresentativity of samples of two materials from food industry. Comput Meth Appl Mech Eng 195(33-36):3960-3982. doi:10.1016/j.cma.2005.07.022

37. Pelissou C, Baccou J, Monerie Y, Perales F (2009) Determination of the size of the representative volume element for random quasi-brittle composites. Int J Solid Struct 46(14-15):2842-2855. doi:10.1016/j.ijsolstr.2009.03.015

38. Lazzarin P, Berto $F(2005)$ Some expressions for the strain energy in a finite volume surrounding the root of blunt v-notches. Int J Fract 135(1-4):161-185

39. Echlin MP, Pollock TM (2013) A statistical sampling approach for measurement of fracture toughness parameters in a 4330 steel by 3-D femtosecond laser-based tomography. Acta Mater 61(15):5791-5799. doi:10.1016/j.actamat.2013.06.023

40. Taylor JR (1997) An Introduction to error analysis: the study of uncertainties in physical measurements. A series of books in physics. University Science Books, Herndon. http://books.google.com/books?id=giFQcZub80oC

41. Groeber M, Ghosh S, Uchic MD, Dimiduk DM (2007) Developing a robust 3-D characterization-representation framework for modeling polycrystalline materials. JOM 59(9):32-36

42. Przybyla C, McDowell D (2012) Microstructure-sensitive extreme-value probabilities of high-cycle fatigue for surface vs. subsurface crack formation in duplex ti-6al-4v. Acta Mater 60(1):293-305

43. Jha S, Caton M, Larsen J (2007) A new paradigm of fatigue variability behavior and implications for life prediction. Mater Sci Eng 468:23-32

\section{doi:10.1186/s40192-014-0021-9}

Cite this article as: Echlin et al:: Three-dimensional sampling of material structure for property modeling and design. Integrating Materials and Manufacturing Innovation 2014 3:21. 